\title{
Proceeding
}

Supplementary Issue: Spring Conferences of Sports Science. Costa Blanca Sports Science Events, 19-20 June 2020. Alicante, Spain.

\section{A study on psychological training of eSports using digital games: Focusing on rhythm game}

\author{
GOICHI HAGIWARA ${ }^{1}$, DAISUKE AKIYAMA², RYOUSUKE FURUKADO ${ }^{3}$, SHUNICHI TAKESHITA ${ }^{1}$ \\ ${ }^{1}$ Faculty of Physical Education, Department of Sports Humanities and Applied Social Science, National \\ Institute of Fitness and Sports in Kanoya, Kanoya-shi, Japan \\ ${ }^{2}$ Faculty of Human Science, Department of Sport Science and Health, Kyushu Sangyo University, Fukuoka, \\ Japan \\ ${ }^{3}$ Faculty of Engineering, Nishinippon Institute of Technology, Fukuoka, Japan
}

\begin{abstract}
Incidentally, several studies have mentioned that digital games or video games have a positive impact on psychological skills (Green, et al., 2003; Pessoa, 2009; Anguera, et al., 2013). Thus, this study aimed to examine the psychological skills focusing on selective attention by comparing the power change in the beta wave band of the EEG during game play and the results of the trail making test (TMT) conducted before and after. Participants were ten male college students who usually play game for at least one hour per day, five days per week (age: $M=21.90 \pm 1.96$ ). This study adopted "Osu!" that is a free online rhythm game as an experimental task, because several articles mentioned that professional gamers usually use "Osu!" as a warming up or practice before gaming (Webb, 2019; Carpenter, 2019). Thus, "Osu!" is assumed to have the effect of enhancing the players' psychological skills. In addition, the TMT part B is adopted to measure attention to speed, motor speed, visual screening, mental flexibility, perseverance, interference affinity and reaction inhibition. As a result of comparing change in biological attention extracted from the EEG during game play or baseline measurement, there is a significant difference in selective attentional state between baseline and while game play. In comparison of TMT indicated that processing speed in after rhythm game was tended to be faster than before play. Thus, this study might be indicated that rhythm game would be the one of the psychological or cognitive training tool for eSports.

Keywords: EEG; Gamification; eSports; Distal game; Rhythm game; Osu!.

Cite this article as:

Hagiwara, G., Akiyama, D., Furukado, R. \& Takeshita, S. (2020). A study on psychological training of eSports using digital games: Focusing on rhythm game. Journal of Human Sport and Exercise, 15(3proc), S495-S503. doi:https://doi.org/10.14198//hse.2020.15.Proc3.03

Corresponding author. Faculty of Physical Education, Department of Sports Humanities and Applied Social Science, National Institute of Fitness and Sports in Kanoya, Kanoya-shi, Kagoshima, Japan.

E-mail: hagiwara-g@nifs-k.ac.jp

Supplementary Issue: Spring Conferences of Sports Science. Costa Blanca Sports Science Events, 19-20 June 2020. Alicante, Spain.

JOURNAL OF HUMAN SPORT \& EXERCISE ISSN 1988-5202

(c) Faculty of Education. University of Alicante

doi:10.14198/jhse.2020.15.Proc3.03
\end{abstract}




\section{INTRODUCTION}

eSports involves the enactment of video games as spectator-driven sport, carried out through promotional activities; broadcasting infrastructures; the socioeconomic organization of teams, tournaments, and leagues; and the embodied performances of players themselves (Taylor, 2016; Reitmen, et al., 2020), and it is phenomenon that has grown dramatically over the past decade (Toth et al., 2019). Sport involves the display of elite athletes' physical and psychological skill in sports competition for entertainment aims in general (Campbell, et al., 2018). On the other hand, eSports' athletes seem to rely more on psychological skills for success rather than motor skills that traditional sports athletes rely on (Himmelstein, et al., 2017). Thus, training of psychological skills for eSports is significant to promote its entertainment and it is necessary to pursue whether to improve psychological skills for eSports athletes.

Incidentally, several studies have mentioned that digital games have a positive impact on psychological skills (Green \& Bavelier, 2012, Pessoa, 2009; Anguera, et al., 2013). Green and Bavelier (2012) found that the digital game play group, which had been playing the game for 6 months or more at least 4 days a week for at least 1 hour a day indicated a significant improvement in the results of the attentional function test. In addition, Hagiwara et al. (2020) examined the relationship between cognitive skill and selective attention extracted from electroencephalograph (EEG) before and after eSports activity, and the result demonstrated that cognitive skill (procession speed) improved after eSports activity, and also selective attention (beta wave band power ratio) appeared during eSports play. Moreover, Lim et al. (2019) measured the EEG of college students while playing the League of Legend, which is a global eSports title, and found that the beta wave band power ratio in the frontal lobe was significantly improved. From the above studies, it is shown that eSports play itself enhances the cognitive function and the psychological skills.

By the way, if a training method that improves processing speed can be established, it may be possible to further increase the value of eSports athletes. According to Özçetin et al. (2019), their study compared processing speed between experts and non-experts for eSports by using the Trail Making Test (TMT), and the result indicated experts had significantly faster processing speed than non-experts. In addition, Sousa et al. (2020) examined processing speed by using the TMT for eSports athletes, and the results indicated the processing speed after the play was significantly faster than before the play. Thus, it indicated that processing speed is one of the factors required for eSports athletes, and it might be necessary to establish a psychological training method that enhances processing speed.

Recently, several professional eSports athletes mentioned that they are usually use rhythm game before practice and competition for mentally or physically setting up their skills (Webb, 2019; Carpenter, 2019). For example, Tyler Ninja Blevins who is the most well-known professional eSports athlete for shooting game uses "Osu!" as a warmup or practice before play (Webb, 2019). "Osu!" (ppy pty Ltd., Australia) is a free online rhythm game that a player taps a series of circles to the beat of the music, occasionally dragging a ball or rotating a spinner on the screen as well. Bégel et al. (2017) conducted a review study to verify the beneficial effects of rhythm games, and study mentioned that training using rhythm games has a positive effect on cognitive function and physical rhythm perception. However, there is no scientific evidence has not been validated and needs to be validated for use by eSports athletes as a training tool.

Thus, this study aimed to examine the potential values of rhythm game as a psychological skill's training method for eSports athletes. For achieving the purpose, this study focuses on selective attention extracted from EEG and processing speed measuring by TMT. 


\section{METHODS}

\section{Participants}

Participants were ten male college students who usually play game for at least one hour per day, five days per week (age: $M=21.90 \pm 1.96$ ). They belonged to the collegiate eSports team. The research team informed the purpose of the study for participants and gave instructions on the experiment prior to participation. Data and informed consent were obtained from the participants. Participation was entirely voluntary.

\section{Procedure}

The experimental procedure is as follows (Figure 1). First, participants were asked to put on an electroencephalograph (EEG) device and measured EEG for two minutes to investigate their baseline degree of arousal before the gaming task. Subsequently, they conducted the Trail Making Test (TMT) evaluating their processing speed on baseline. Later, they conducted the gaming task. Gaming task was the rhythm game. This study adopted "Osu!" that is a free online rhythm game as an experimental task, because several articles mentioned that professional gamers usually use "Osu!" as a warming up or practice before gaming (Webb, 2019; Carpenter, 2019). Participants played ten minutes. While plying "Osu!", participants measured EEG to investigate their degree of selective attention, and they conducted the TMT after the gaming task. Selective attention was measured by calculating the power ratio in the beta wave band extracted from the EEG. Detailed method will be introduced in the following section.

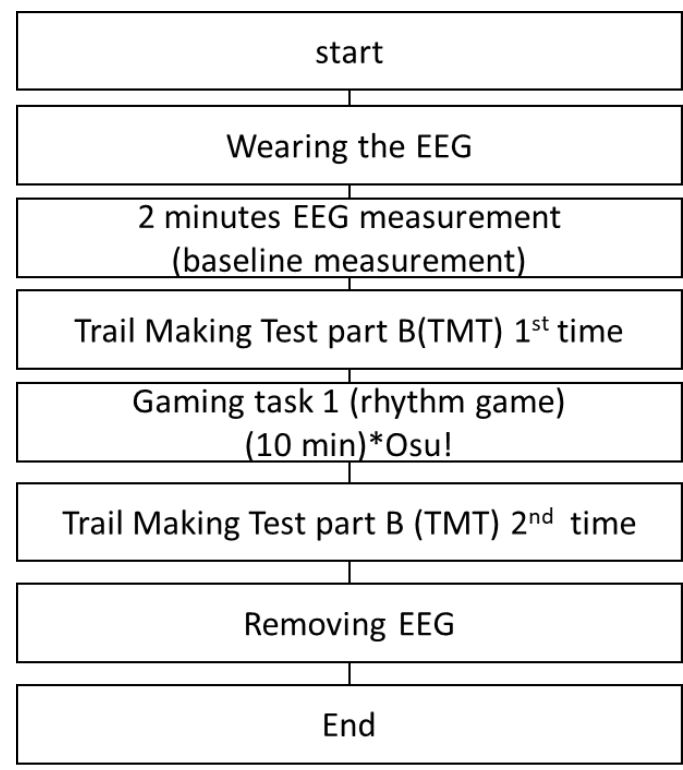

Figure 1. Procedure of experiment.

\section{Evaluation of processing speed}

For evaluating processing speed, the TMT was adopted in this study, because several studies mentioned that the TMT is best conceptualized as reflecting a combination of several cognitive functions (Newby et al., 1983; Kortte et al., 2002). The TMT measures attention to speed, visual screening and reaction inhibition. It consists of two parts in which the participant is required to draw lines on a page connecting twenty-five dots consecutively as quickly as possible, and this study adopted the Part B that participants must draw lines alternating between numbers and letters in order (Kortte et al., 2002). Performance of the TMT is assessed 
by time taken to complete the tasks. The Part B were especially associated with processing speed on complex tasks and cognitive flexibility (Kortte et al., 2002), thus, in this study, the Part B was judged to be suitable for measuring the effects of eSports, which the situation changes drastically.

\section{Psychological evaluation from EEG}

A psychological evaluation in the form of an EEG was conducted to assess the selective attention state of participants. In addition, the EEG measurement was used in this study because it is more easily measured in a daily living environment compared to other brain activity measurements. We adopted a simple band-type EEG device (NeuroSky Co., Ltd., Japan) that only measures the front polar area 1 (Fp1) lobe as defined by the international 10-20 system (Figure 2).

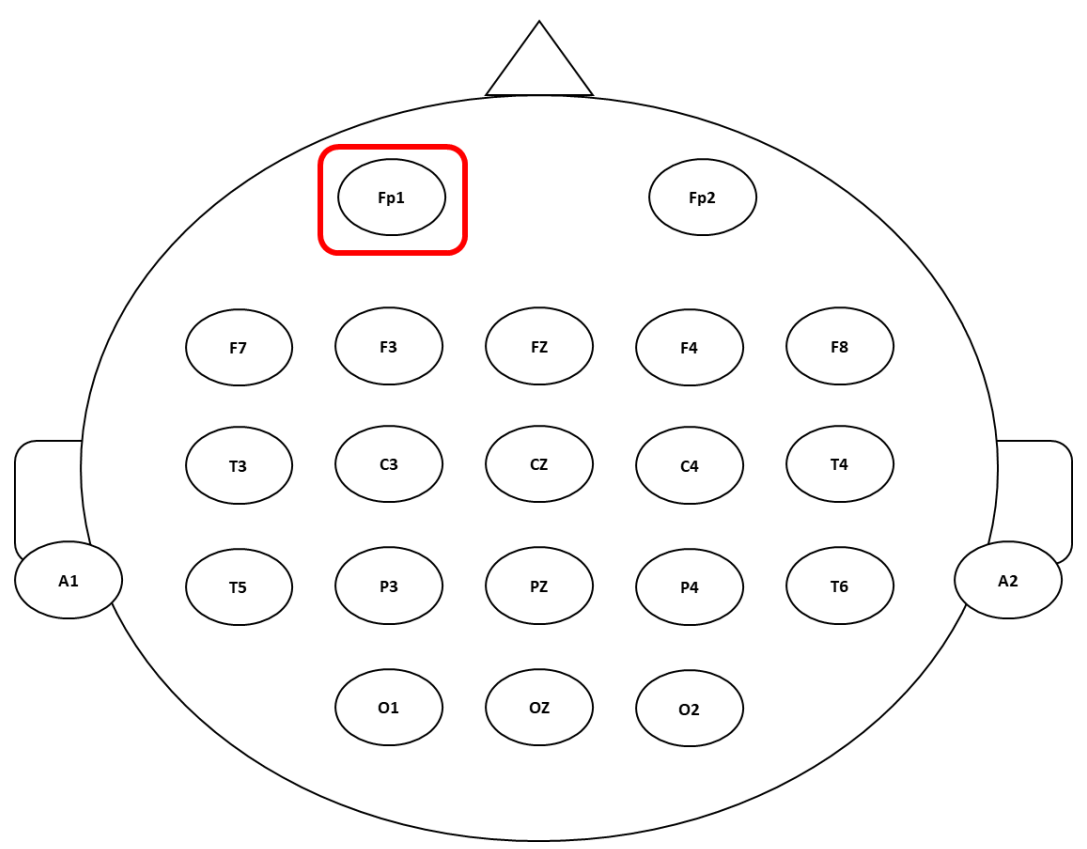

Figure 2. Measurement points of the international 10-20 system.

Since Fp1 is located on the left frontal lobe, there is no need to worry about the possible noise interference caused by hair. The EEG obtained from Fp1 has been found to be suitable for obtaining data on people's psychological condition (Mitsukura, 2016; Hotta et al., 2017). Thus, this study adopted Fp1 to estimate selective attention level.

The electrical activity in the brain is often used as an objective evaluation index that employs biological data. Brainwaves are generally classified into four types according to their frequency range $(0.5-4 \mathrm{~Hz}$ : delta waves; 4-8 Hz: theta waves; 8-13 Hz: alpha waves; 13-40 Hz: beta waves) (Okubo, 2018), and generally the beta waves are indicated that the frequency band of the EEG was extracted during selective attention in the human active states (Firel, 2007; Heinrich et al., 2007). Therefore, this study focused on the beta wave band, and calculated the integrated value of the power based on the power spectrum obtained by the fast Fourier transform. In addition, the ratio of beta wave activity in the total power (occurrence ratio) was calculated in order to determine the relative activity of brain waves in the beta wave band. Thereby, the occurrence ratio of the selective attention degree is calculated. In addition, the Sports KANSEl (Littlesoftware Inc., Japan) was set so that the occurrence ration of the beta wave band was defined as the selective attention level, and the 
value was easily displayed as a value from 0 to 100 . Hagiwara et al. (2020) posited that the selective attention level output by the Sports KANSEI was related with processing speed of the cognitive skill test.

The basic concept of Sports KANSEl that it calculates the power ratio between the beta wave bands. The potential difference obtained from the electrodes on the forehead and earlobe of the left Fp1 is amplified by the circuit inside the measuring instrument, digitized at $512 \mathrm{samples} / \mathrm{sec}$, and subjected to the Hanning window processing. The power spectral analysis was then conducted using the fast Fourier transform. EEG data are analysed every second by the fast Fourier transform, and the amplitude spectra can be obtained in the frequency range of 1-64 Hz. Thus, this study obtained delta, theta, alpha and beta waves. From the obtained power spectrum, the sum of each power in each frequency band was calculated after, and the ratio included in the total of the total power is shown as a relative numerical value. However, the sum of the power of each frequency cannot be used because the amplitude of each frequency band is different. Therefore, we took the average value of the power of each frequency band and obtained the representative value of that frequency band. The calculation method used the following formula as a standard for analysis.

The average Px of the $x$-wave power was calculated by formula (1), where $V f$ is the power of the EEG at the frequency $f[\mathrm{~Hz}]$. In this study, since the beta wave band $(13 \mathrm{~Hz}$ to $40 \mathrm{~Hz})$ is handled, when $x=\beta$, it becomes $(13,40)[\mathrm{Hz}]$, and the numerical value is applied to (Fxmax - Fxmin) in formula (1). Next, the sum of power averages (Psum) in each frequency band is calculated by formula (2). The ratio (Rx) included in the total power of the beta wave band was calculated in formula (3).

$$
\begin{aligned}
& P_{x}=\sum_{f=F_{\text {min }}^{x}}^{F_{\text {max }}^{x}} V_{f} /\left(F_{\max }^{x}-F_{\text {min }}^{x}+1\right) \\
& P_{\text {sum }}=P_{\delta}+P_{\theta}+P_{\alpha}+P_{\beta} \\
& R_{x}=P_{x} / P_{\text {sum }}
\end{aligned}
$$

Based on the above calculation method, the Sports KANSEI normalized the power ratio that can be taken in the beta band to the value of $0-100$.

\section{Analysis}

In order to verify the change of processing speed by playing the rhythm game, the task performance time of TMT was compared before and after the game play. t-test was used to compare before and after. In addition, t-test was adopted to compare the average degree of selective attention between two minutes' baseline measurement and ten minutes while playing rhythm game. IBM SPSS Statistics 25.0 software was used for the analysis.

\section{RESULTS AND DISCUSSION}

As a result of comparing the processing speed by TMT before and after playing rhythm game, there was significant difference $(t=1.17, p<.01)$. After playing rhythm game, processing speed $(M=38.53 \pm 12.80$ seconds) was significantly faster than before ( $M=41.33 \pm 18.12$ seconds) (Figure 3$)$. In addition, the result of comparing the average degree of selective attention extracted from EEG between two minutes' baseline measurement and ten minutes while playing rhythm game, it was tended to be significantly different $(t=3.76$, 
$p<.1)$. It was shown that the degree of selective attention during the rhythm game $(M=57.00 \pm 8.33)$ tended to be significantly higher than that of the baseline $(M=47.50 \pm 8.22)$ (Figure 4).

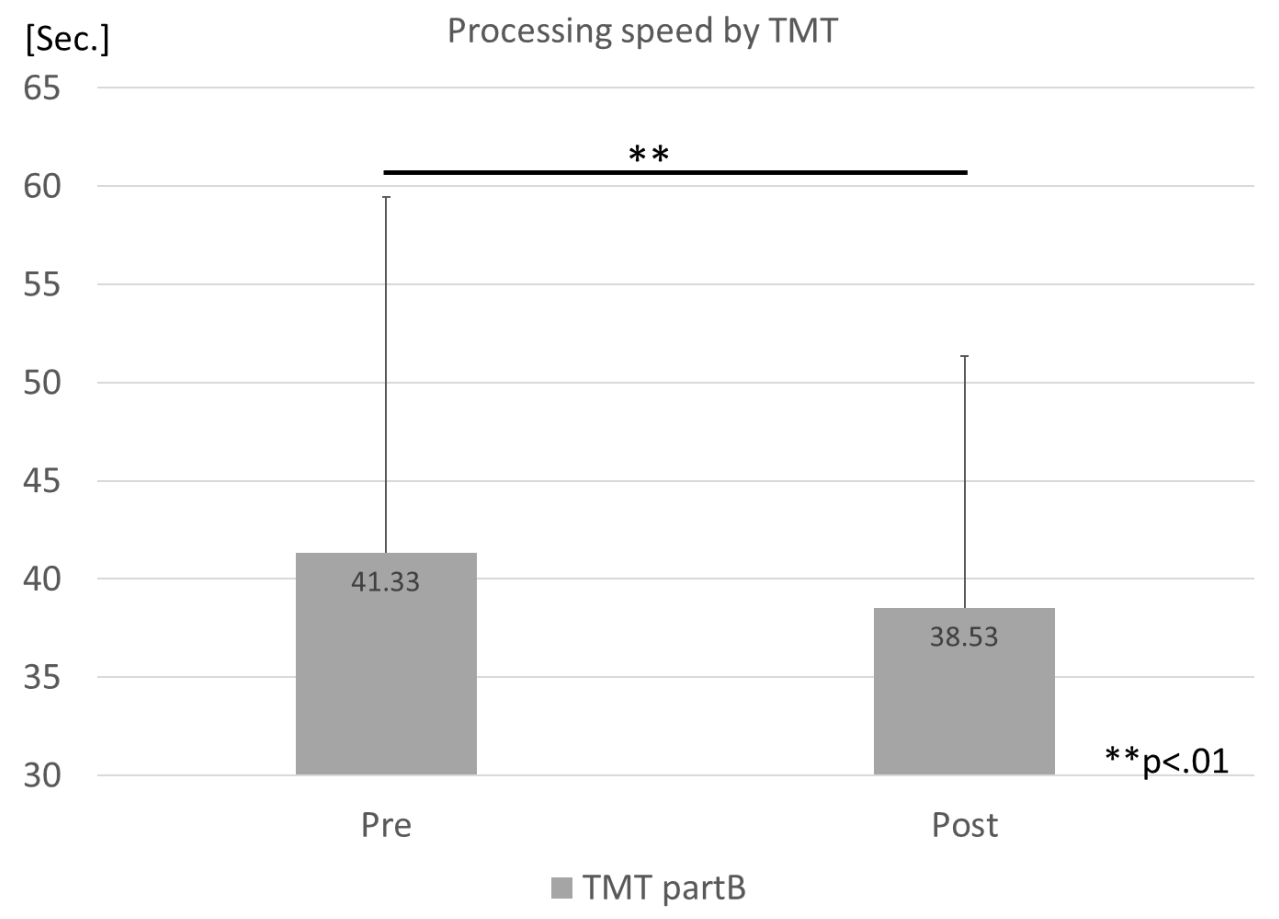

Figure 3. Comparing the processing speed by TMT pre and post-test.

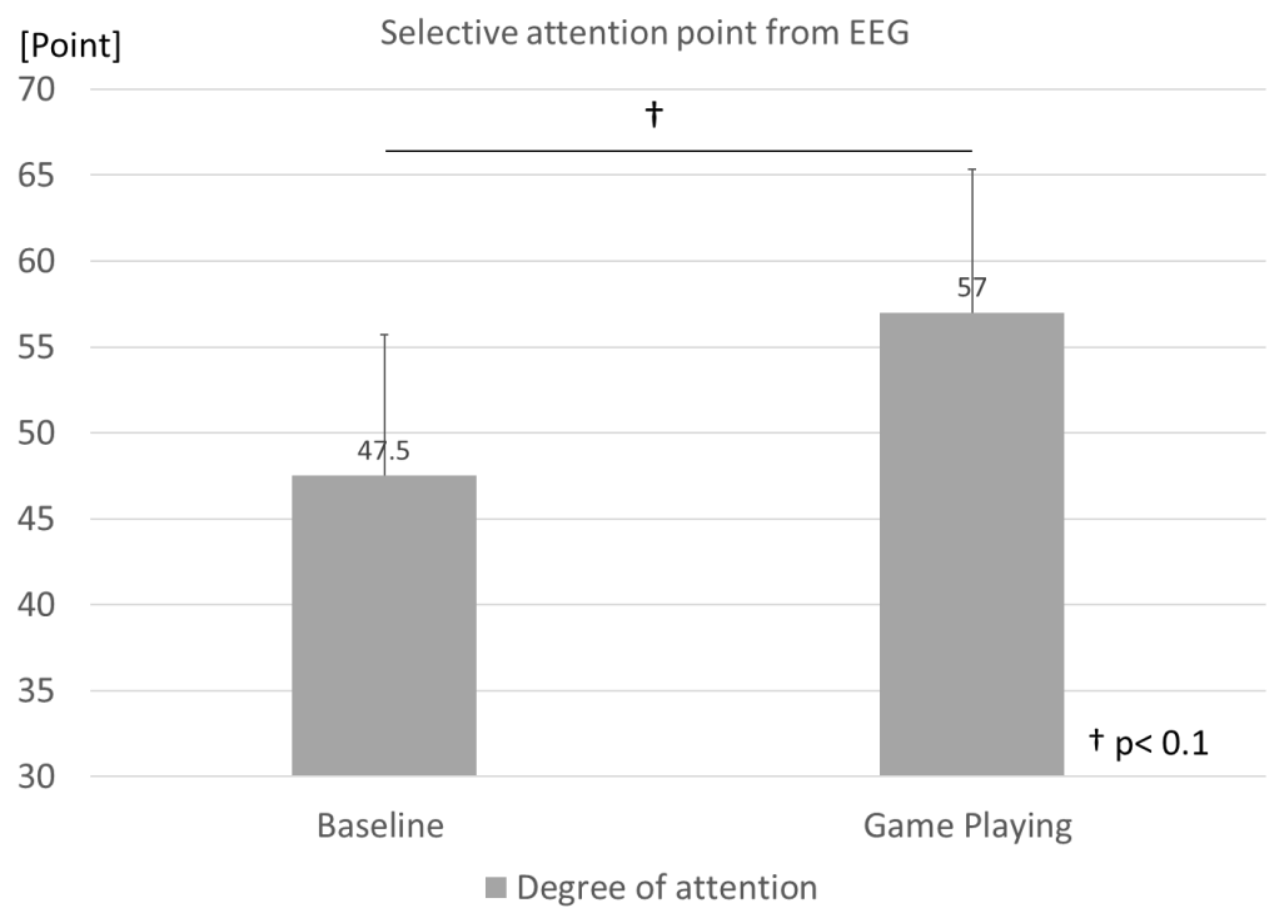

Figure 4. Comparing the average degree of selective attention in baseline and while playing "Osu!". 
From the above results, it was found that playing a rhythm game increases the processing speed for complicated tasks, and this study also found that the degree of selective attention increased during the rhythm game. Bégel et al. (2017) conducted systematic reviews about music games to verify the beneficial effects of rhythm training and found that training using rhythm games has a positive effect on cognitive function and human movement. In addition, they mentioned that rhythm games might be adopted to cognitive training. Özçetin et al. (2019) compared processing speed between experts and non-experts for eSports by using the TMT, and they found that experts had significantly faster processing speed than non-experts. Also, Sousa et al. (2020) found that the processing speed after the playing eSports was significantly faster than before. Thus, it indicated that processing speed is one of the factors required for eSports athletes. The results of this study also indicated that the processing speed by TMT was increased by playing "Osu!". Especially, TMT is best conceptualized as reflecting a combination of several cognitive functions and it associated with processing speed on complex tasks and cognitive flexibility (Newby et al., 1983; Kortte et al., 2002) Therefore, it might be confirmed that eSports players could even improve several cognitive skills by playing "Osu!" as a cognitive skill training tool.

As we mentioned that several professional eSports players usually use rhythm game like "Osu!" before practice and competition for mentally or physically setting up their skills (Webb, 2019). Also, some professional players use "Osu!" as a psychological training for increasing their attentional skills or concentration (Carpenter, 2019). This study demonstrated that selective attention state extracted from EEG was increased while playing "Osu!". Incidentally, several studies have mentioned that digital games have a positive impact on psychological skills (Green, et al., 2003; Pessoa, 2009; Anguera, et al., 2013). For example, Hagiwara et al. (2020) found that selective attention state extracted from EEG was significantly increased during playing the car game. Moreover, Lim et al. (2019) found that the beta wave band power ratio in the frontal lobe was significantly improved while playing the Multiplayer Online Battle Arena (MOVA) games. From these studies, it is shown that eSports play itself enhances the psychological skills such as selective attention. This study adopted "Osu!" to investigate that how rhythm games affect to psychological skills by measuring selective attention state for collegiate eSports gamers, and the result of this study might be explained rhythm game such as "Osu!" would be an essential training tool for increasing psychological skills. However, there are limitations to this study. We conducted the experiments on ten collegiate eSports gamers belong to the eSports club, but it is necessary to expand the sample size in order to generalize this study. It is thus necessary that future studies be conducted with more participants. In addition, although this study conducted the experiment in a one time, it is recognized that selective attention state may change depending on the condition of the participants on that day. Therefore, it is suggested that conducting a longitudinal experiment on the same participants might shed more accurate findings on the matter.

In conclusion, this study aimed to examine the potential values of rhythm game as a psychological skill's training method for eSports athletes, and found that playing a rhythm game such as "Osu!" increases the cognitive skills and the psychological skills. We hope that rhythm games would become one of the psychological trainings tool for eSports players in the future.

\section{REFERENCES}

Anguera, J.A., Boccanfuso, J., Rintoul, J.L., Al-Hashimi, O., Faraji, F., Janowich, J., Kong, E., Larraburo, Y., Rolle, C., Johnston, E., \& Gazzaley, A. (2013). Video game training enhances cognitive control in older adults. Nature, 501, 97-101. https://doi.org/10.1038/nature12486 
Bégel, V., Di Loreto, I., Seilles, A., \& Dalla Bella, S. (2017). Music Games: Potential Application and Considerations for Rhythmic Training. Front. Hum. Neuroscience, 11, 273. https://doi.org/10.3389/fnhum.2017.00273

Campbell, M. J., Toth, A. J., Moran, A. P., Kowal, M., \& Exton, C. (2018). eSports: a new window on neurocognitive expertise? Prog. Brain Res. 240, 161-174. https://doi.org/10.1016/bs.pbr.2018.09.006

Carpenter, N. (2019). Gamers with godlike reflexes are racing to break world records in this rhythm game. PC Gamer. https://www.pcgamer.com/two-teens-are-on-a-crazy-world-record-race-in-extremelychallenging-rhythm-game-osul (2020, June 15)

Friel P. N. (2007). EEG biofeedback in the treatment of attention deficit hyperactivity disorder. Altern. Med. Rev., 12(2), 146-151.

Green, C. S., \& Bavelier, D. (2012). Learning, attentional control, and action video games. Curr. Biol. 22, R197-R206. https://doi.org/10.1016/i.cub.2012.02.012

Hagiwara, G., Kawahara, I. \& Kihara, S. (2020). An attempt to verify the positive effects of eSports: focusing on concentration and cognitive skill. J. Jpn. Soc. Sports Ind., 30, (in press).

Heinrich, H., Gevensleben, H., \& Strehl, U. (2007). Annotation: neurofeedback - train your brain to train behaviour. J. Child. Psychol. Psychiatry., 48(1), 3-16. https://doi.org/10.1111/j.1469$\underline{7610.2006 .01665 . X}$

Himmelstein, D., Liu, Y., \& Shapiro, J. L. (2017). An exploration of mental skills among competitive league of legend players. Int. J. Gaming Comput. Mediated Simul. 9, 1-21. https://doi.org/10.4018/ijgcms.2017040101

Hotta, M. \& Kohata, Y. (2017). The evaluation of usability of EC site using electroencephalogram (EEG). Proc. 19th Jpn. Soc. Kansei Eng., 1-5.

Kortte, K. B., Horner, M. D., \& Windham, W. K. (2002). The trail making test, Part B: Cognitive flexibility or ability to maintain set? Appl. Neuropsychol., 9(2), 106-109. https://doi.org/10.1207/s15324826an0902_5

Lim, S.; Yeo, M. \& Yoon, G. (2019). Comparison between Concentration and Immersion Based on EEG Analysis. Sensors, 19, 1669. https://doi.org/10.3390/s19071669

Mitsukura, Y. (2016). KANSEI Analyzing by EEG. J. Inst. Electr. Eng. Jpn., 136, 687-690. https://doi.org/10.1541/ieeijournal.136.687

Newby, R. F., Hallenbeck, C. E., \& Embretson, S. (1983). Confirmatory factor analysis of four general neuropsychological models with a modified Halstead-Reitan battery. J. Clin. Neuropsychol., 5(2), 115-133. https://doi.org/10.1080/01688638308401159

Okubo, T., Tamamaru, K. \& Koshimizu, S. (2018). Development of the Impression Detection System by using a Portable EEG Device for Tourist Impression Analysis. Trans. Jpn. Soc. Kansei Eng., 17, 285291. https://doi.org/10.5057/jiske.tjske-d-17-00082

Özçetin, M., Gümüştaş, F., Çağ, Y., Gökbay, İ. Z., \& Özmel, A. (2019). The relationships between video game experience and cognitive abilities in adolescents. Neuropsychiatr. Dis. Treat., 15, 1171-1180. https://doi.org/10.2147/ndt.s206271

Pessoa, L. (2009). How do emotion and motivation direct executive control? Trends Cog. Sci. 13(4), $160-$ 166. https://doi.org/10.1016/i.tics.2009.01.006

Reitmen, J. G., Anderson-Coto, M. J., Wu, M., Lee, J. S. \& Steinkuehler, C. (2020). Esports research: a literature review. Game Cult., 15, 32-50. https://doi.org/10.1177/1555412019840892

Sousa, A. \& Ahmad, S. L., Hassan, T., Yuen, K., Douris, P., Zwibel, H., \& DiFrancisco-Donoghue, J. (2020). Physiological and Cognitive Functions Following a Discrete Session of Competitive Esports Gaming. Front. Psychol., 11. https://doi.org/10.3389/fpsyg.2020.01030 
Taylor, N. (2016). Play to the camera: video ethnography, spectatorship, and e-sports. Convergence, 22, 115-130. https://doi.org/10.1177/1354856515580282

Toth, A., Kowall, M. \& Campbell, M. J. (2019). The color-word stroop task does not differentiate cognitive inhibition ability among esports gamers of varying expertise. Front. Psychol., 10, Article2852. https://doi.org/10.3389/fpsyg.2019.02852

Webb, K. (2019). Professional gamers like Ninja use this music game to practice their aim and improve their mouse skills - here's how you can play for free. Business Insider. Retrieved from https://www.businessinsider.com/osu-game-pro-gamers-practice-aim-improve-mouse-skillsesports-2019-8 (2020, June 15).

\section{(c)}

This work is licensed under a Attribution-NonCommercial-NoDerivatives 4.0 International (CC BY-NC-ND 4.0). 